\title{
APORTACIONES AL CONOCIMIENTO DEL GENERO EQUISETUM L. II. E. X LITORALE RUPR.
}

\author{
J. IRANZO, C. PRADA, M. VILAR \& A.E. SALVO
}

RESUAEN: Se realiza una descripción detallada de los caracteres morfológicos y anatomo-histológicos de Equisetum $x$ litorale Rupr. (E. arvense $x$ E. fluviatile).

RESUME: On réalise una description détaillée des caractères anatomo-histologiques et morphologiques de Equisetu $x$ litorale Rupr. (E. arvense $x$ E. fluviale ).

\section{INTRODUCCION}

Durante una campaña realizada a los Puertos de Somiedo (León) en 1984 recolectamos en comunidades de Pinguiculo grandiflorae-Caricetum lepidocarpae Rivas-Martínez et al. 1984 (Scheuzerio-Caricetea fuscae) varias especies del género Equisetum (E. variegatum Schleicher, E. fluviatile L., E. arvense L. y E. palustre L.), además del híbrido que nos ocupa, del cual existe el siguiente testimonio de herbario: León: Subida al Puerto de Somiedo, 28.VI.1984, Salvo et al. (MGC).

A pesar de que Page (1982) señala que E. x litorale es el hibrido de este género más frecuente, tan sólo hemos encontrado una cita del mismo para España. Esta se debe a Ruiz de Azúa (1928), quien afirma haberlo encontrado en Tolosa (Guipúzcoa). Aunque la descripción morfológica y anatómica que acompaña a la cita coincide con la descripción del taxon, sin embargo las ilustraciones de los cortes de los vástagos y ramas que adjunta corresponden a E. fluviatile. Desgraciadamente en todo el material estudiado recolectado por Ruiz de Azúa no hemos encontrado ningún pliego correspondiente a este híbrido.

Equisetum x litorale Kühlew. ex Rupr. in Beitr. Pflanzenk. Russ. Reiches, 4: 91 (1845) (= E. arvense $\times$ E. fluviatile) fue visto por vez primera por Kühlewein en el litoral arenoso marino de las proximidades de Oranienbaum (Rusia). Cuando Ruprecht describe esta planta, señala ya su posición morfológica intermedia entre E. arvense y E. fluviatile, y así en su protólogo incluye tan sólo las diferencias y semejanzas respecto a estas dos especies. Muchos autores posteriores redescribieron el taxon bajo diferentes sinónimos. Lasch (1857) sería el primer autor que reconociera el origen hibridógeno del mismo, destacándolo bajo el epíteto específico E. arvensi-limosi. Milde (1867) realizó un profundo estudio morfológico y anatómico de este híbrido, dejando bien patente la posición intermedia de los caracteres respecto 
a los dos progenitores. Ducket \& Page (1975) observaron que si bien el intergrado es manifiesto, sin embargo el híbrido es extremadamente variable y en ocasiones puede ser muy semejante a cualquiera de los padres, relacionando estos grados extremos con el hábitat colonizado. Así, afirman que en hábitats secos es más próximo a E. arvense, mientras que en hábitats húmedos lo es a E. fluviatile. Estos autores señalan finalmente que a veces la diferenciación morfológica no es suficiente para la confirmación diagnóstica y que es necesario efectuar análisis anatómicos.

\section{DESCRIPCION}

Rizoma. El rizoma de E. x litorale presenta una coloración castaño-oscura a negra brillante. De los entrenudos parten raices relativamente cortas y cubiertas por pequeños y numerosos pelos. Las partes jóvenes del rizoma presentan aun vainas de hojas, no portando raices.

El corte transversal del entrenudo del rizoma (Fig. 1) presenta una sección más o menos circular, no diferenciándose por tanto ni carenas ni valléculas, como ocurre en el tallo aéreo y en el rizoma joven.

La epidermis del rizoma está formada por células prismáticas, cuyas paredes celulares se encuentran bastante engrosadas e impregnadas totalmente de suberina; suberificación que alcanza también a las células externas del parénquima cortical subyacente.

El parénquima cortical presenta dos tipos de células. Las periféricas forman una banda continua de varios estratos por debajo de la epidermis, caracterizándose por presentar engrosamientos colenquimatosos en sus paredes celulares y numerosos amiloplastos en sus citoplasmas. Las otras, que forman el resto del parénquima del rizoma y que se dispone por debajo de las anteriores, entre las lagunas corticales y alrededor de los haces vasculares, presentan un lumen celular aparentemente vacío donde existen vacuolas muy voluminosas.

En este híbrido es imposible distinguir el parénquima cortical del medular, ya que la endodermis, provista de Banda de Caspary, rodea a cada uno de los haces vaculares y las células parenquimáticas interlagunares son iguales o muy semejantes a las células parenquimáticas perivasculares, por lo que preferimos aludir a "parénquima general" para referirnos en este caso concreto a todo el tejido parenquimático indiferenciado.

El rizoma adulto presenta grandes lagunas corticales, que ocupan hasta el $60 \%$ de la sección, decreciendo notablemente este porcentaje en el rizoma joven. Precisamente a este hecho atribuimos las diferencias entre nuestras observaciones y las de Milde (1867).

La laguna central del rizoma bien desarrollado (Fig. 2) es bastante más pequeña que las lagunas corticales, estando limitada por el parénquima perivascular.

Los haces vasculares del rizoma, lo mismo que los del tallo aéreo, presentan una endodermis individual, con una Banda de Caspary, que los rodea totalmente. Por debajo de la endodermis se encuentra una fila de células que forman el periciclo y que como las células del parénquima cortical externo contienen amiloplastos.

Los haces vasculares presentan protoxilema y metaxilema funcionales, a diferencia de lo observado en E. fluviatile en donde apenas se desarrolla metaxilema, recordando en este aspecto a E. arvense.

Vástagos aéreos. Los vástagos aéreos de E. x litorale alcanzan, en los ejemplares estudiados, hasta $50 \mathrm{~cm}$ de longitud; son verdes y 
poseen ramificaciones que comienzan hacia la mitad del tallo, decreciendo éstas progresivamente hacia el ápice, que es indiviso. Los entrenudos presentan alrededor de 10 carenas poco pronunciadas, número este intermedio entre las de E. fluviatile (12 a 20) y las de E. ar-vense ( 4 a 14) (Fig. 3 A, B y C).

La epidermis de E. $x$ litorale presenta numerosas mamelas y pílulas siliceas dispuestas formando pequeñas crestas transversales, semejantes a las de E. arvense, si bien las mamelas epidérmicas del híbrido son más pequeñas y romas que las de aquel progenitor. Las características propias de los estomas (número, morfología, etc.) se establecen en un rango intermedio entre los parentales. Así, mientras que E. fluviatile presenta sus estomas dispuestos en 12-14 filas por toda la superficie vallecular y $E$. arvense presenta dos dobles filas (a lo sumo dos triples), en el híbrido suelen observarse 4-6 dobles filas en cada vallécula.

La organización tisular subepidérmica del entrenudo de E. x litorale recuerda bastante a la E. arvense. Así, el colénquima en el híbrido se dispone tanto carinal como vallecularmente, tal como se presenta en aquel parental. Sin embargo, mientras que el colénquima carinal en esta especie se restringe únicamente a la protuberancia carinal, en E. $x$ litorale el colénquima carinal se prolonga más o menos hacia los haces vasculares, lo que provoca que el clorénquima a nivel de las carenas se encuentre disociado, en oposición a E. arvense con clorénquima continuo. Por otra parte, el colénquima vallecular es menos importante en el híbrido que en E. arvense, y puede faltar en los tallos jóvenes, sobre todo en los artejos terminales del tallo, disponiéndose entonces el clorénquima de una forma semejante a como lo hace en E. fluviatile. Aunque la estructuración del clorénquima ya se comentó anteriormente, es necesario destacar que en el híbrido es más reducido que en sus parentales y limita internamente con el parénquima general.

Hacia el interior del colénquima y clorénquima, y limitando las lagunas corticales, los haces vasculares y la laguna central, se encuentra un "parénquima general" formado por células homogéneas.

El resto de la sección del tallo está ocupada por las lagunas corticales y la laguna central, siendo, a la inversa que en el rizoma, las primeras más pequeñas que la segunda. Las lagunas corticales presentan un contorno irregular, con el diámetro tangencial casi doble que el diámetro radial. Este carácter es bastante bueno para diferenciar al híbrido de los parentales, ya que en E. arvense el diámetro tangencial suele ser la mitad del radial y en E. fluviatile, aunque es corriente que no aparezcan lagunas corticales en los tallos delgados ni en la parte superior de muchos tallos, cuando las hay (base del tallo), entonces los diámetros tangenciales superan ampliamente a los radiales.

La laguna central es bastante grande ocupando aproximadamente un tercio del diámetro total del tallo, por tanto más similar a E. arvense que a E. fluviatile, que presenta una enorme laguna central (Fig. 4).

Los haces vasculares del tallo aéreo presentan una endodermis individual para cada uno de los haces. El metaxilema en el tallo aéreo está más desarrollado que el protoxilema, que al parecer deja de ser funcional cuando crecen los entrenudos y queda únicamente como resto no funcional en alguna de las lagunas vasculares.

Vainas de micrófilas. Las vainas de micrófilas que rodean los nudos de los vástagos aéreos de E. $x$ litorale son semejantes a las de E. arvense por ser más largas que anchas y por no estar tan estrechamente aplicadas contra el tallo como en E. fluviatile; al igual 
que en aquel parental, presentan células áncoras o anquirocitos en la comisura de las hojas, sobre todo en el segmento medio de las vainas; este tipo de células no aparecen en las vainas de E. fluviatile (Fig. 5). Por otra parte, los dientes de las vainas del híbrido están con frecuencia soldados lateralmente de dos en dos, y no presentan -l surco dorsal que poseen los de E. arvense.

El parénquima clorofílico y el haz conductor ocupan casi la totalidad del mesófilo de la hoja. Apenas se observan células colenquimatosas por debajo de la costilla foliar.

La epidermis abaxial aparece densamente cubierta de mamelas y pilulas, presentando las primeras una peculiarmorfología en semiesferas con el ápice aplanado.

Ramificaciones laterales. En E. $x$ litorale las ramas laterales, muy parecidas a las de E. arvense, son simples con el primer entrenudo más largo que la vaina caulinar adyacente, o raramente igualándola, trianguladas o tetranguladas, con los valles débilmente surcados, lo que hace que no sean tan marcadamente en $V$ como en E. arvense $\mathrm{ni}$ en $\mathrm{U}$ como en E. fluviatile. Las vainas rameales tienen los dientes agudos, estrechos, con una prominente costilla dorsal, ápice oscuro y estrecho margen membranoso.

La organización general de estas ramas laterales (Fig. 6) recuerda mucho a la del tallo aéreo, si bien pueden establecerse algunas claras diferencias. Así la laguna central es proporcionalmente menor que la del tallo aéreo y el córtex está ocupado por un parénquima clorofílico muy desarrollado. Las lagunas corticales pueden estar presentes o no, y su tamaño es muy variable cuando existen. El colénquima de las ramas laterales se sitúa como en el tallo, en las carenas y en las valléculas, pero es mucho más pobre que el del tallo aéreo.

\section{AGRADECIMIENTOS}

Queremos expresar nuestra sincera gratitud a los Doctores Díaz-González, Penas y Puente del Departamento de Botánica de la Universidad de León por su colaboración y asesoramiento.

\section{BIBLIOGRAFIA}

DUCKET, J.G. \& PAGE, C.N. -1975- Equisetum. In Stace, C.A. "Hybridization and the Flora of British Isles": 99-103. Academic Press.

HAUKE, R.L. -1978- A taxonomic monograph of Equisetum subgenus Equisetum. Nova Hedwigia 30: 385-451.

IRANZO, J., VILAR, M. \& SALVO, A.E. -1985- Aportaciones al conocimientc del género Equisetum L. I. Descripción de los microcaracteres de E. palustre L. Acta Bot. Malacitana, 10: 33-40.

LASCH, W. -1857- Aufzählung der in des Provinz Bradenburg, besonders in der Gedend um Driesen wildwaschsenden Bastard-Pflanzen. Bot. Zeit. (Berlin), 15: 505 .

MILDE, J. -1867- Monographia Equisetorum. Nova Acta Peop.-Carol., 32(2).

PAGE, C.N. -1982- The ferns of Britain and Ireland. Cambridge University Press. 
RUIZ DE AZUA, J. -1928- Nota preliminar acerca de los equisetos españoles. Bol. R. Soc. Esp. Hist. Natural, 27: 229-235.

Dirección de los autores: J. IRANZO, M. VILAR, Departamento de Botánica, Facultad de Farmacia, Universidad de Valencia, 46010 VALENCIA. C. PRADA, Departamento de Botánica, Facultad de Biología, Universidad Complutense, 28040 MADRID. A.E. SALVO, Departamento de Botánica, Facultad de Ciencias, Universidad de Málaga, Apartado 59, 29080 MALAGA. 


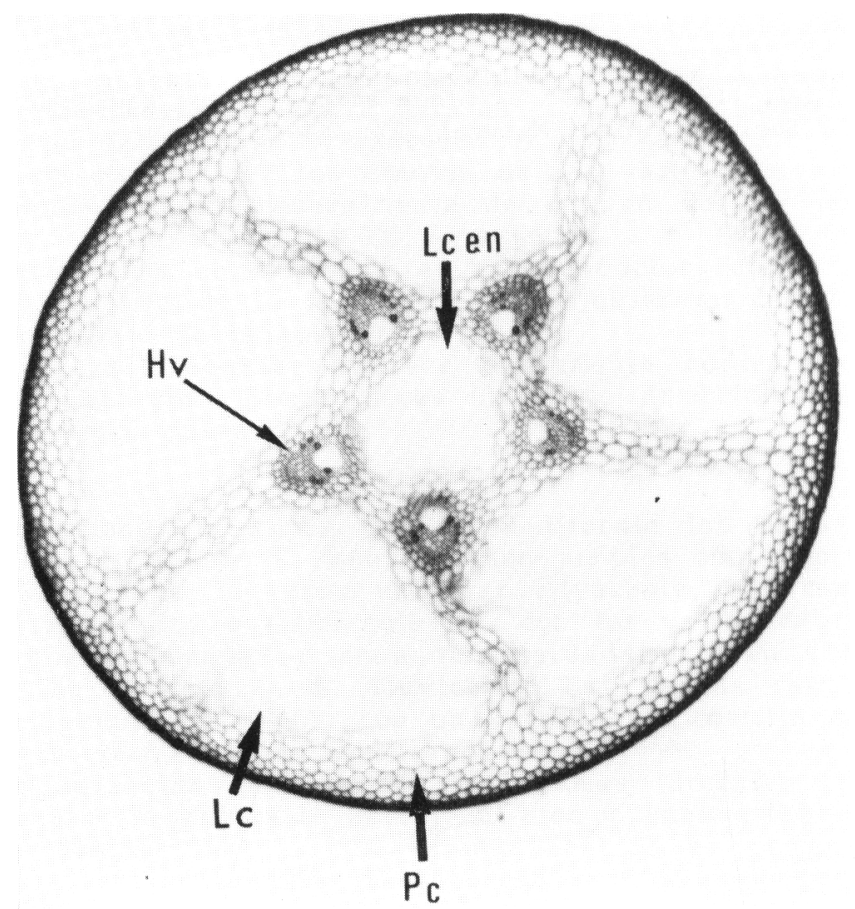

Figura 1.- Sección transversal del entrenudo del rizoma de E. x litorale.

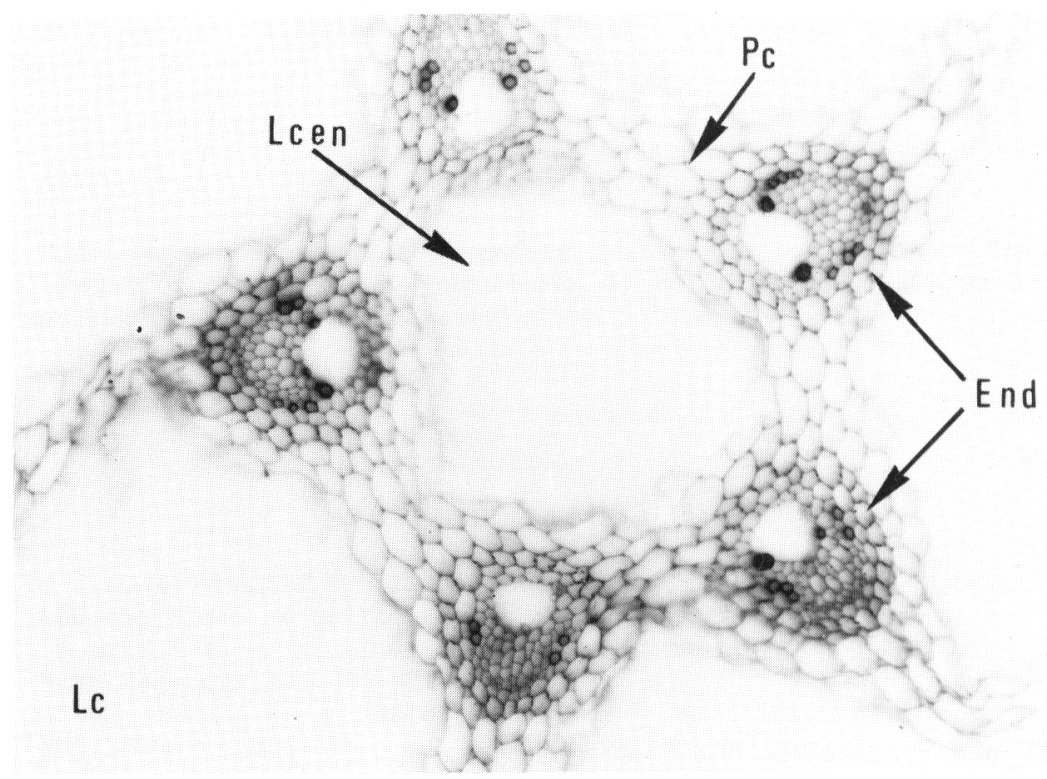

Figura 2.- Detalle de los haces vasculares en los entrenudos del rizoma de E. x litorale.

Clave de abreviaturas de las figuras.- An: Anquirocitos. Bc: Banda de Caspari. Cl: Clorénquima. Eex: Epidermis exterior. Ein: Epidermis interior. End: Endodermis. Hv: Haz vascular. Lc: Laguna cortical. Lcen: Laguna central. Lm: Laguna medular. Lv: laguna vascular. mx: Metaxilema. Pc: Perénquima cortical. Per: Periciclo. Pm: Parénquima medular. pX: Protoxilema. 

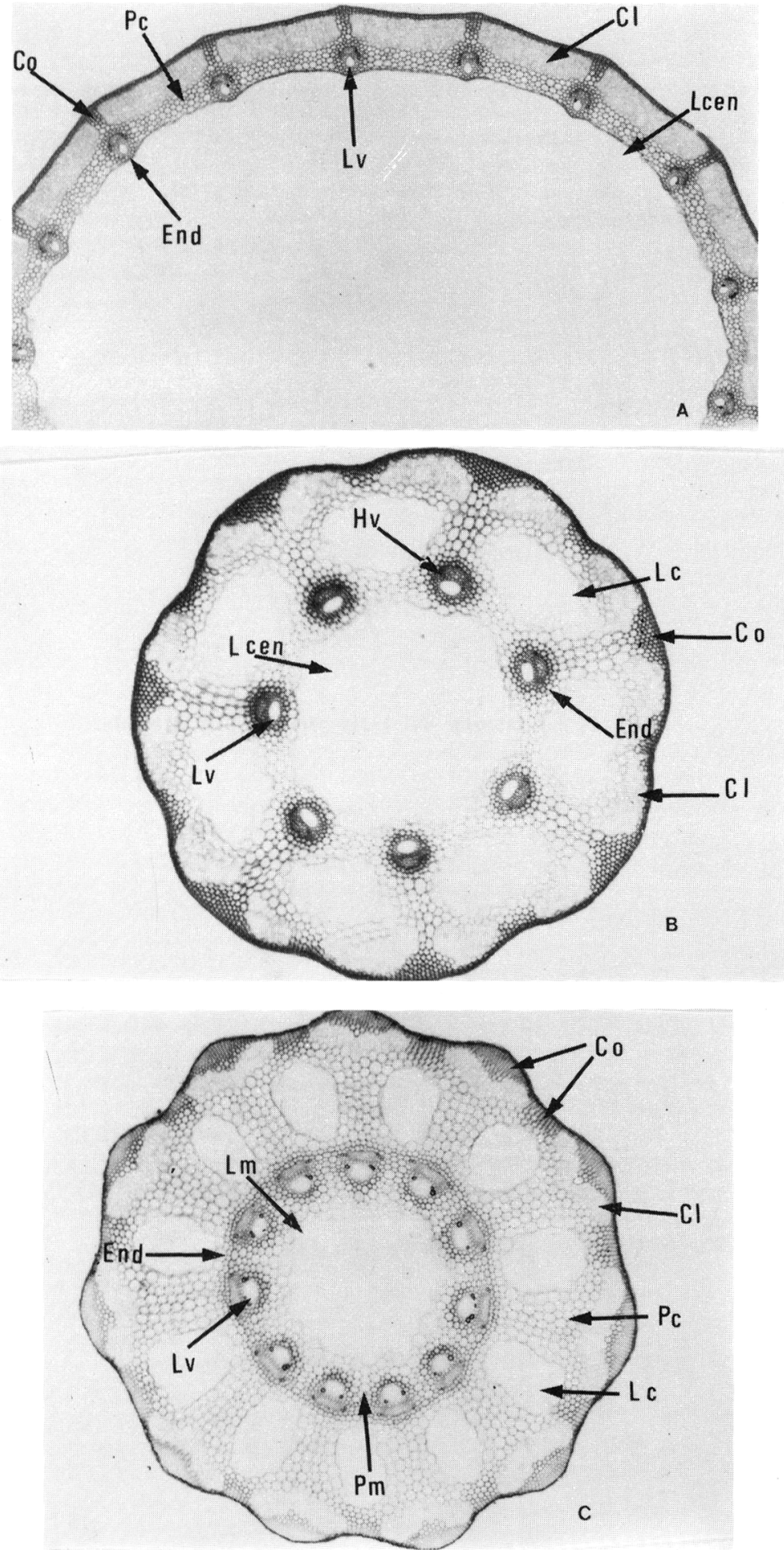

Figura 3.- Secciones transversales de los entrenudos de los tallos aéreos de: A: E. fluviatile; B: E. x litorale; C: E. arvense. 


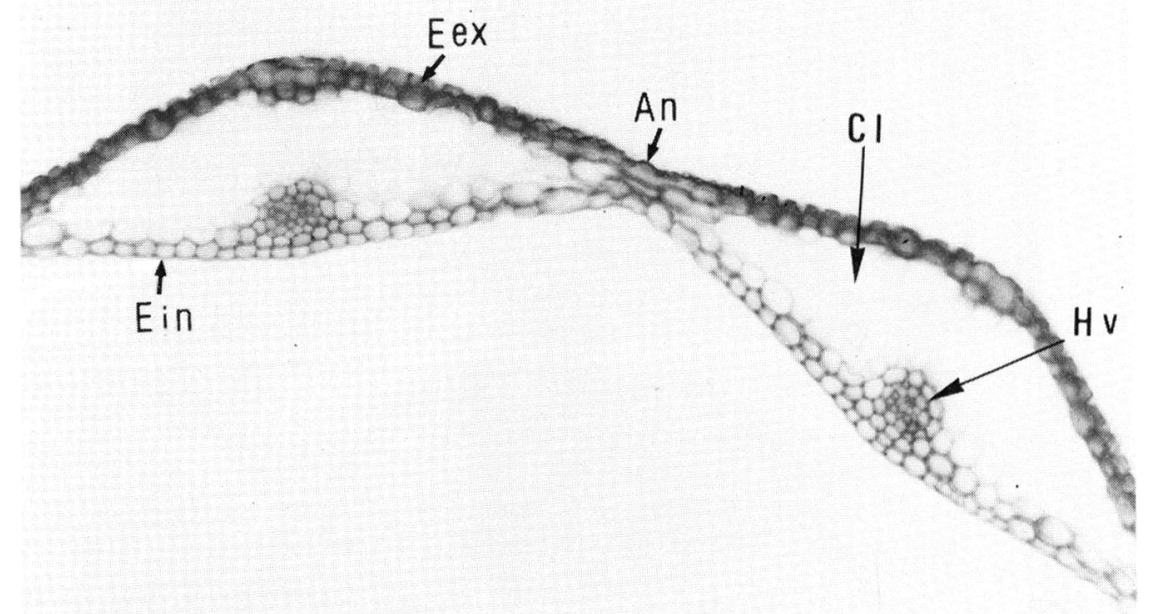

Figura 4.- Haz vascular del tallo aéreo de E. x litorale.

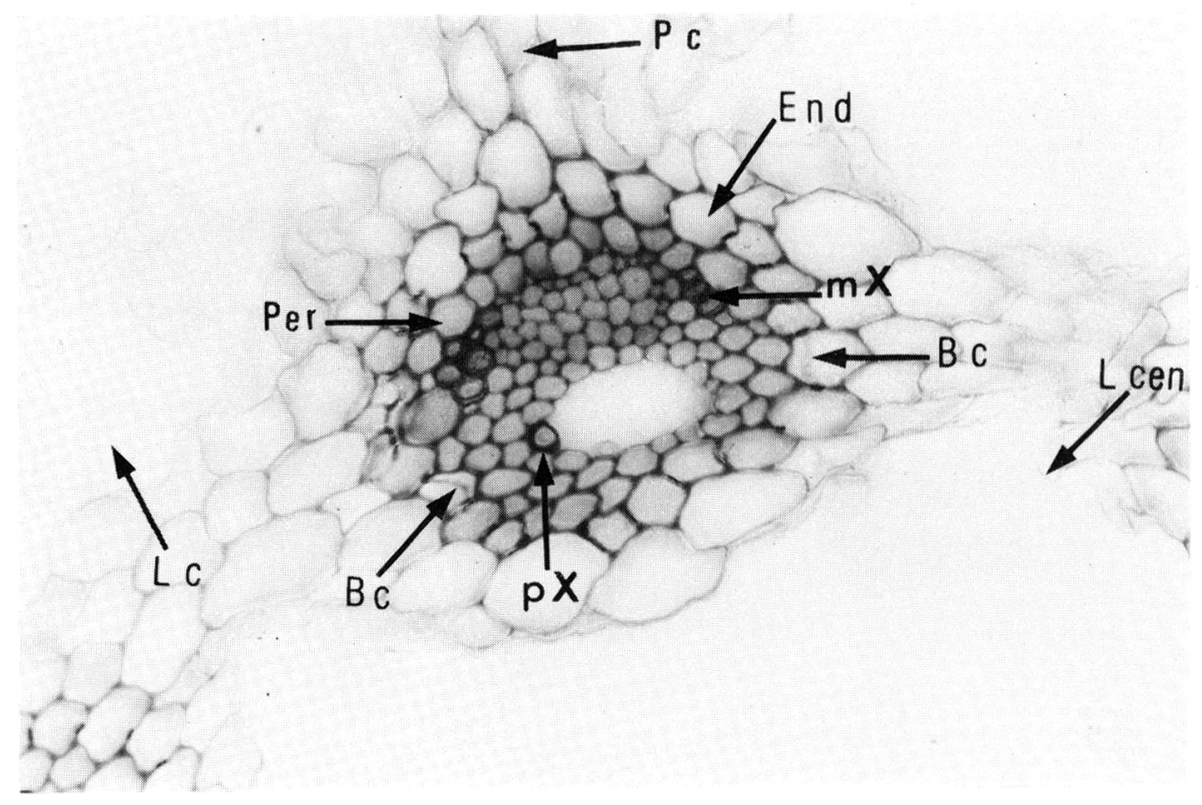

Figura 5.- Detalle de la sección transversal de la vaina de E. x litorale. 

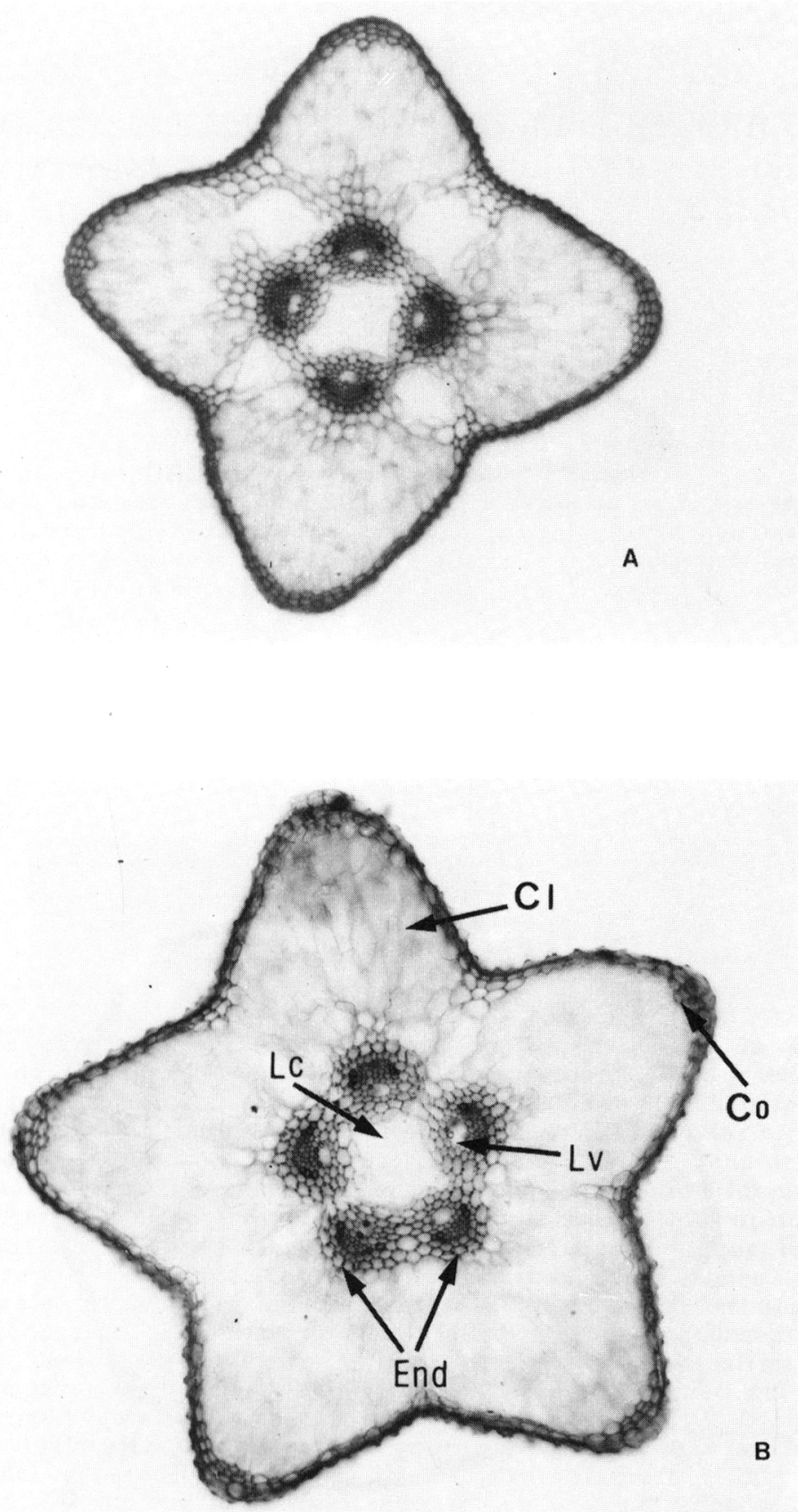

Figura 6(1).- Secciones transversales de las ramificaciones de ( $A$ y $B$ ) E. $x$ litorale. 

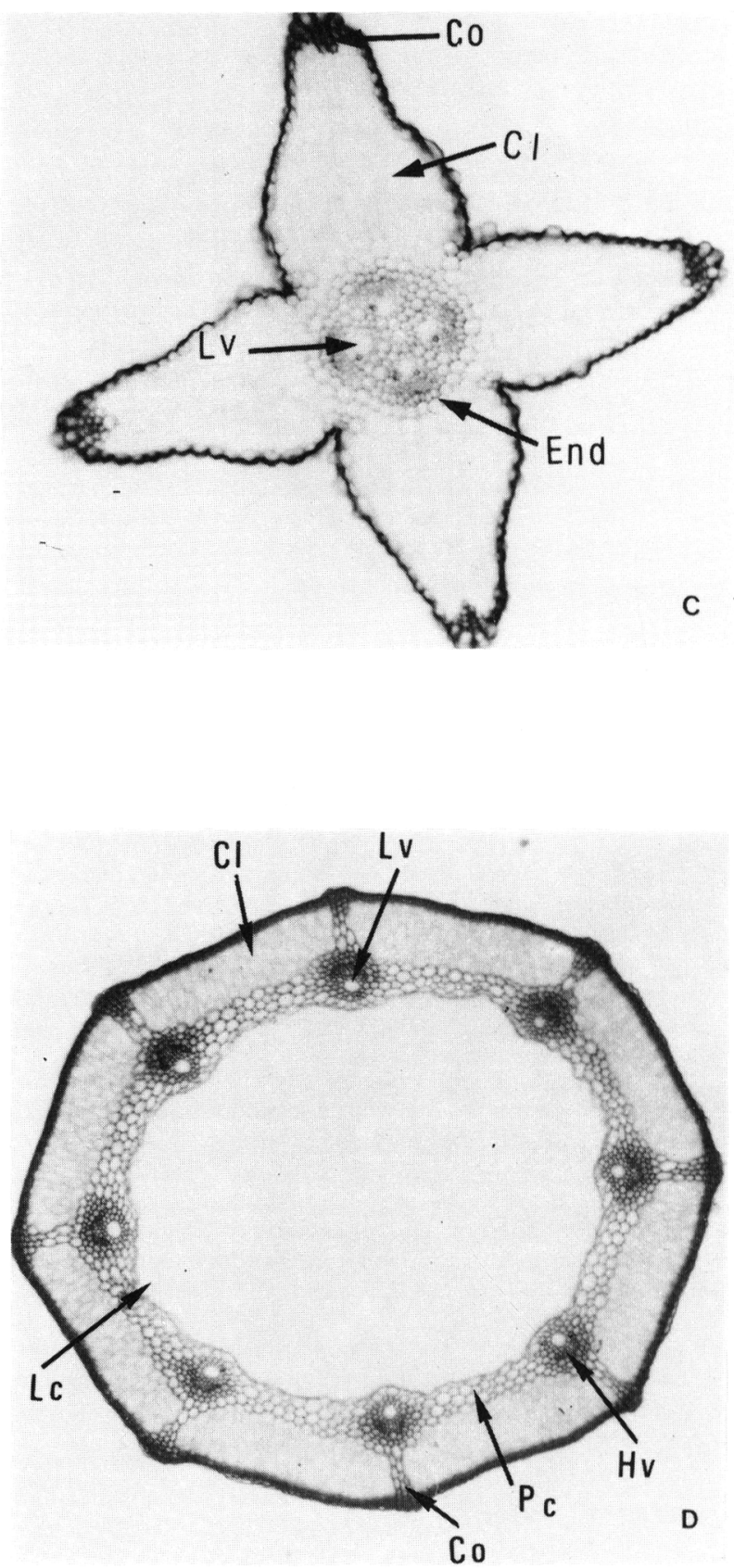

Figura 6(2).- Secciones transversales de las ramificaciones de (C) E. arvense, (D) E. fluviatile. 